\title{
RESEARCH
}

\section{The path to diagnosis for persons with ALS in Nova Scotia}

\author{
J Colin Boyd MSc', Terri Cooper ${ }^{2}$, Brian D O'Brien, MD MSc ${ }^{3}$ \\ ${ }^{1}$ Class of 2018, Faculty of Medicine, Dalhousie University \\ ${ }^{2}$ Amyotrophic Lateral Sclerosis Society of New Brunswick \\ ${ }^{3}$ Departments of Medicine and Community Health and Epidemiology, Dalhousie University
}

\section{Abstract}

Purpose: The purpose of this study was to examine the route to diagnosis of persons with Amyotrophic Lateral Sclerosis (ALS) in Nova Scotia.

Methods: Nova Scotia clients of the ALS Society of New Brunswick and Nova Scotia described their perspective on their route to a firm diagnosis of ALS, including processes that worked effectively as well as barriers to expedited care. Clients were selected from all who had been diagnosed in the previous two years and who were able and willing to participate in the interview.

Results: Fifteen interviews were completed. From two to 42 months elapsed between the time of initial symptoms and firm diagnosis of ALS being made. The path to diagnosis was $\leq 6$ months for five individuals and was $\geq 18$ months for five others. Mean duration of time elapsed between the first symptoms and the firm diagnosis was 14.4 months with a median of 11 months.

Conclusions: Persons with ALS in Nova Scotia have similar paths to diagnosis compared to those in other countries. Considerable delays occurred in persons with initial bulbar symptoms and in those cases where ALS was not considered a diagnostic possibility early in the course, due to lack of provider awareness or atypical initial symptoms and signs.

$\mathrm{T}$ There are between 20-25 new cases of amyotrophic lateral sclerosis (ALS) from Nova Scotia registered annually with the ALS Society of New Brunswick and Nova Scotia (ALSNBNS). Although it is an uncommon disease, ALS has an overwhelming impact on the individuals with the disease and their families. A firm diagnosis is a prerequisite to obtaining services from sources funded by charitable organizations such as the ALSNBNS. Further, some private insurers may require a firm diagnosis before providing coverage for assistive devices, pharmaceuticals and nursing care. Many ALSNBNS clients report inordinate delays during the diagnostic process, causing great delays in obtaining required support services.

ALS is the most common form of progressive motor neuron disease and one of the most devastating neurological disorders. It is characterized by degeneration of lower and upper motor neurons in the central nervous system with bulbar involvement in about 20 percent of cases. Selective and progressive motor neuron cell death results in muscle weakness, wasting, and eventually mortality, which typically results from respiratory paralysis, a median of three to five years after diagnosis. In 2003, the crude and age adjusted incidences in Nova Scotia were 2.24 cases and 2.13 cases per 100,000 respectively. ${ }^{1}$ These rates are similar to those found in other western countries.

In recent years, persons with ALS (PALS) have secured an improved quality of life through respiratory and nutritional support as well as improved communication and mobility devices. However, some of these services are not provided by the publiclyfunded health care system in Canada. ALSNBNS is a charitable organization with no public funding which provides significant support to PALS but not until a definitive diagnosis is made. Therefore, delays in diagnosis of ALS may lead to delays in the provision of needed assistance.

PALS in Nova Scotia report misdiagnosis, diagnostic testing, and specialist referral as contributors to delayed diagnosis of ALS. Delays in diagnosis may increase uncertainty on the part of patients and their families, delay supportive treatment and end-of-life planning, and result in missed opportunities to enter clinical trials and unnecessary testing and procedures..$^{2-4}$

This study was designed to examine the route to diagnosis for PALS in Nova Scotia from the individual's perspective. Many of the issues identified above are likely to be encountered in this province given that there is only one major referral centre in Nova Scotia, community services are varied throughout the province, and the distribution of physicians with expertise in this disorder is uneven.

\section{Methods}

The study team consisted of an epidemiologist, a staff member from client services at the ALS Society, and a 


\begin{tabular}{ccl} 
Participant & Months to Diagnosis & Reason for Delay \\
\hline A & 10 & Clinical complexity \\
B & 32 & Primary care delay \\
C & 18 & Primary care delay \\
D & 42 & Clinical complexity, primary care delay \\
E & 15 & Clinical complexity \\
F & 11 & Clinical complexity \\
G & 22 & Clinical complexity, primary care delay, delay in secondary care pathway \\
H & 2 & No delay \\
I & 6 & Clinical complexity \\
J & 24 & Clinical complexity, primary care delay \\
K & 4 & No delay \\
L & 13 & Clinical complexity \\
M & 3 & No delay \\
N & 8 & Clinical complexity \\
O & 6 & No delay \\
\hline
\end{tabular}

Table 1. Participant summary data.

research assistant. Registered clients were approached to enter this study when the client services staff believed they were ready to be invited. The initial invitation contained an outline of the study including its rationale and outline of the methods. If the invitation to participate was accepted, the client was sent a detailed consent form and a visit by the team was arranged with the client and any others the client invited (e.g., caregivers). At the visit, consent was obtained in writing before the client and caregivers were interviewed. If the client was not able to communicate verbally, the client could indicate consent to the accompanying person who then signed on the client's behalf. If the client wished, the caregiver could provide information. In this situation, written consent was also obtained from the caregiver. If unable to communicate verbally, the client could use a communication device. The interview was initially open-ended and prompted by a request to describe how the diagnostic process unfolded. Necessary specific follow-up questions to clarify timelines, including the time of first symptoms, the initial and subsequent clinical care, and the time of firm diagnosis were asked. The interview guide is attached online as Annex A. An audio recording was made of the interview and notes were made to facilitate analysis.

The interview material was analyzed independently by two researchers (JCB, BDO) who developed themes that described both the good and bad experiences that clients had on their path to diagnosis. Disagreements were resolved by consensus. Timelines from symptoms to diagnosis were created for each client and mean and median times were calculated.

Sample size was determined by the principle of saturation as well as the availability and willingness of the clients to participate. As this was a qualitative study, the sample size was not determined by the number of participants required to make statistical generalizations. Instead, the goal was to explore what kinds of issues arose during the diagnostic process. Accordingly, the number of interviews to be conducted was dependent on the point at which the likelihood of new significant issues arising balanced against the expenditure and time required for further interviews.

The protocol was approved by the Dalhousie University Health Sciences Research Ethics Board.

\section{Results}

Information was obtained from fifteen PALS. A summary of each participant's total time to diagnosis and relevant delays is available in Table 1. Fourteen of the PALS were interviewed directly and the spouse of one recently deceased was also interviewed. Eight lived in urban areas (defined as Halifax, Dartmouth, Bedford, Sackville, or Sydney, Nova Scotia) and seven lived in rural areas. From two to 42 months elapsed between the time of initial symptoms to a firm diagnosis of ALS being made. The path to diagnosis was $\leq 6$ months for five individuals and was $\geq 18$ months for five others. Mean duration of time elapsing between the first 
symptoms and a firm diagnosis was 14.4 months with a median of 11 months.

Four individuals felt that there was no delay and that they received a definitive diagnosis promptly. In nine cases, the clinical situation was quite complex due to atypical symptoms and complicated by inconclusive initial investigations. In five cases, the initial primary care provider did not recognize that the patient might have ALS, causing either delayed referral or referral to an incorrect specialist. In three cases, delays resulted from multiple referrals to other specialties and other diagnoses before a neurologist became involved. Typically, these patients presented with bulbar symptoms only. Of interest, seven of the fifteen individuals in this study were quite convinced they had ALS sometime before the formal diagnosis.

\section{Discussion}

This study of 15 PALS in Nova Scotia showed that the median time from initially seeking medical care to diagnosis was eleven months, with a range from two to forty-two months. Two-thirds of cases received a firm diagnosis within eighteen months. There were no differences in time to diagnosis between those who lived in urban areas and those who lived in rural ones.

The diagnosis of ALS can be challenging as there is no positive diagnostic test. It is diagnosed primarily on clinical grounds, supported by the absence of electrophysiological or neuroimaging evidence of another disease that might explain the upper and lower motor neuron signs. ${ }^{5}$ Thus persons with symptoms and signs of neurologic disease may require both time to have the syndrome evolve as well as undergo many investigations before a definitive ALS diagnosis can be made. From the patient's perspective, this can be both prolonged and frustrating.

In a study of US Veterans enrolled from 1957 to 2005 in a national registry, the time to first diagnosis was defined as the time from onset of new progressive weakness to the time that ALS was recorded as the diagnosis, as documented in the health record. ${ }^{2} 1099$ registry participants with ALS had a median time to first diagnosis of ten months. The time to diagnosis was least for those with bulbar onset and greatest for lower limb onset. Limitations noted by the study's authors included the predominately male gender of the participants as well as their frequent occupational exposure to wartime conditions.

Mitchell and co-workers studied 640 patients of the Preston Motor Neuron Disease Care Centre in the United Kingdom diagnosed between 1989 and 2008. ${ }^{3}$ They report a median time from first symptoms to diagnosis of 0.95 years and suggest three themes in explaining delays to diagnoses. In cases where symptoms and signs were atypical, or initial investigations were inconclusive, the diagnosis was often difficult. If the general practitioner first seeing the patient did not recognize that ALS was a possible diagnosis, the patient was often observed over several months or referred to an inappropriate specialist for investigation. Delay in secondary care pathways occurred where some patients experienced multiple referrals before seeing a neurologist. These other specialists often failed to recognize that the patient may have ALS and referred patients to specialist departments other than neurology. Such patients often underwent lengthy investigations by other specialists before neurological referral.

In a thorough qualitative study of the causes of delay in diagnosis in England, there were several themes elaborated in patient interviews. ${ }^{6}$ Primary care physicians did not always perceive an urgency to make specialist referrals. Referrals were sometimes requested of specialties other than neurology. For instance, some patients were referred for otolaryngology evaluation and the lack of a lesion would sometimes misleadingly reassure the patient. The various delays were often interpreted by patients as a lack of knowledge by treating physicians about ALS.

In a study of 304 clients referred to the Massachusetts General Hospital ALS Clinic, Paganoni et al. noted that those with bulbar onset had a shorter time to diagnosis than those with limb onset. ${ }^{4}$ However, in contrast, those with fasciculations and limb weakness also had shorter times. Conversely, our results suggest a longer time to diagnosis in those with bulbar onset. This may reflect the small number of subjects in our study, where one or two cases could influence the median time to diagnosis unduly. Alternatively, this may reflect the nature of our study population including such aspects as the proportion of clients with a familial form of the disease, rural vs. urban distribution, or the referral patterns and the nature of medical services available locally.

In contrast to the studies discussed above, our study examined the clients' perceptions of the route to diagnosis and their impressions of what worked well and what did not. The health records were not examined as the stimulus for the study was PALS' concerns expressed to the staff of the ALS Society. Further study could incorporate the health records of the primary care physicians and the specialist clinics, which may provide a valuable medical perspective to compare with that of the clients. Because of the delays encountered by PALS, initiatives examining the benefit of education of the public and their primary care providers may be worth exploring. 
Limitations of the study include the small sample; that the information was obtained by interview from the PALS' perspective after the diagnosis was made, which may have allowed a recall bias to influence the result; and that the PALS' health records were not examined.

We conclude that the path to diagnosis in Nova Scotia is similar to that in other countries, both in timing and route. Considerable delays occurred in persons with initial bulbar symptoms and in those cases where ALS was not considered a diagnostic possibility early in the course, due to lack of provider awareness, or atypical initial symptoms and signs.

\section{Acknowledgments}

The study team wishes to acknowledge the encouragement and cooperation received from all the clients and their caregivers and members of the ALS Society of New Brunswick and Nova Scotia, especially the Board Chair, John Forsyth, and the CEO, Kimberly Carter. Support was provided by the ALSNBNS

\section{References}

1. Bonaparte JP, Grant IA, Benstead TJ, Murray TJ, Smith M. ALS incidence in Nova Scotia over a 20 -year-period: a prospective study. Can J Neurol Sci 2007;34(1):69-73.

2. Khishchenko N, Allen KD, Coffman CJ, Kasarskis EJ, Lindquist JH, Morgenlander JC, et al. Time to diagnosis in the National Registry of Veterans with Amyotrophic Lateral Sclerosis. Amyotroph Lateral Scler 2010;11(1-2):125-32.

3. Mitchell JD, Callagher P, Gardham J, Mitchell C, Dixon M, Addison-Jones R, et al. Timelines in the diagnostic evaluation of people with suspected amyotrophic lateral sclerosis (ALS)/motor neuron disease (MND)--a 20-year review: can we do better? Amyotroph Lateral Scler 2010;11(6):537-41.

4. Paganoni S, Macklin E, Lee A, Murphy A, Chang J, Zipf A, et al. Diagnostic timelines and delays in diagnosing amyotrophic lateral sclerosis (ALS). Amyotrophic Lateral Sclerosis \& Frontotemporal Degeneration [serial online]. 2014; 15(5/6): 453-456.

5. Paganoni S, Macklin EA, Lee A, Murphy A, Chang J, Zipf A, et al. Diagnostic timelines and delays in diagnosing amyotrophic lateral sclerosis (ALS). Amyotroph Lateral Scler Frontotemporal Degener 2014;15(5-6):453-6.

6. Brooks BR, Miller RG, Swash M, Munsat TL. El Escorial revisited: revised criteria for the diagnosis of amyotrophic lateral sclerosis. Amyotroph Lateral Scler Other Motor Neuron Disord 2000;1(5):293-9.

7. O'Brien MR, Whitehead B, Jacki BA, Mitchell JD. From symptom onset to a diagnosis of amyotrophic lateral sclerosis/motor neuron disease (ALS/MND): experiences of people with ALS/ MND and family careers - a qualitative study. Amyotroph Lateral Scler 2011;12:97-104 